\title{
Propiedades psicométricas de la Escala de Locus de Control de la Salud en Dios en una muestra de mexicanos con diabetes mellitus tipo 2
}

\section{Psychometric properties of the God Locus of Health Control scale in a sample of mexicans with type 2 diabetes mellitus}

\author{
Vianey Guadalupe Argüelles-Nava a,, Sergio Dominguez-Lara ${ }^{\mathrm{b}}$, \\ Jaime Morales-Romero ${ }^{a}$, Roberto Lagunes-Córdoba ${ }^{c}$ \\ anstituto de Salud Pública, Universidad Veracruzana, México \\ bInstituto de Investigación de Psicología, Universidad de San Martín de Porres, Perú \\ 'Instituto de Investigaciones Psicológicas, Universidad Veracruzana, México
}

\section{Resumen}

En este estudio se obtuvo evidencia de validez y confiabilidad de la escala de Locus de Control de la Salud en Dios (GLHC) en una muestra de 539 adultos mexicanos con diabetes mellitus tipo 2. El análisis de validez se basó en el análisis del contenido de los ítems y de la estructura interna del GLHC. El primer procedimiento estuvo a cargo de seis expertos, quienes evaluaron la pertinencia de la adaptación de los ítems. El segundo procedimiento fue un análisis factorial confirmatorio de grupo múltiple, de acuerdo al sexo y edad, a fin de evaluar la invarianza de medición del GLHC. Finalmente, la confiabilidad fue calculada mediante los coeficientes $\omega$ y $\mathrm{H}$. El análisis factorial confirmatorio demostró una estructura de un solo factor con seis ítems, interpretable para la población de estudio con invarianza de medición por sexo, aunque deben tomarse precauciones al interpretar comparaciones por grupos de edad. La confiabilidad del constructo obtuvo magnitudes elevadas (>.90). Los resultados indican que la GLHC puede brindar información válida y confiable para valorar el locus de control de la salud en Dios para mexicanos con diabetes mellitus tipo 2 .

Palabras clave: Locus de control de la Salud en Dios, validez de constructo, confiabilidad, análisis factorial confirmatorio, diabetes mellitus tipo 2.

Para citar este artículo:

Argüelles-Nava, V., Dominguez-Lara, S., Morales-Romero, J., \& Lagunes-Córdoba, R. (2017). Propiedades psicométricas de la escala de locus de control de la salud en Dios en una muestra de mexicanos con diabetes mellitus tipo 2. Liberabit, 23(2), 259-272. doi: 10.24265/liberabit.2017.v23n2.07

\begin{abstract}
The validity and reliability of the God Locus of Health Control (GLHC) scale were determined in a sample of 539 Mexican adults with type 2 diabetes mellitus. The validity analysis was based on the analysis of the GLHC scale's items content and internal structure. The first procedure was conducted by six experts who evaluated the pertinence of the items adaptation. The second procedure consisted of a multiple-group confirmatory factor analysis by gender and age, aimed to evaluate the GLHC scale's measurement invariance. Finally, reliability was estimated by means of coefficients $\omega$ and $H$. The confirmatory factor analysis demonstrated a six-item unifactorial structure, interpretable for the study population with measurement invariance by gender. Nevertheless, precautions should be taken when interpreting age-group comparisons. The magnitude of the construct reliability was high $(>0.90)$. The results indicate that the GLHC scale may provide valid and reliable information to assess the God Locus of Health Control in Mexicans with type 2 diabetes mellitus.
\end{abstract}

Keywords: God Locus of Health Control, construct validity, reliability, confirmatory factor analysis, type 2 diabetes mellitus.

Este es un artículo Open Access bajo la licencia Creative Commons Atribución-NoComercial-CompartirIgual 4.0

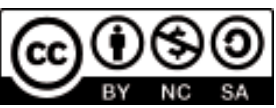




\section{Introducción}

La diabetes mellitus tipo 2 (DM2) es un problema de salud pública de grandes dimensiones en México y produce un alto impacto en la calidad de vida de quienes la padecen, no solo por los síntomas y manifestaciones inherentes a ella, sino por su gran potencial para causar mayor daño a la salud con el paso del tiempo (Hernández-Ávila, Gutiérrez, \& Reynoso-Noverón, 2013). Cifras recientes indican que su prevalencia en adultos de veinte o más años de edad, pasó de 9.2\% en el año 2012 a 9.4\% en el año 2016 (ENSANUT MC, 2016). Sin embargo, uno de los principales problemas vinculados a la aparición de complicaciones asociadas a la DM2 y a un peor estado de salud es precisamente la falta de adherencia al tratamiento (Edelman \& Polonsky, 2017; JiménezCorona \& Aguilar-Salinas, 2013; Shams, Amjad, Kumar, Ahmed, \& Saleem, 2016).

El tratamiento de la DM2 implica cambios significativos en el estilo de vida de quienes la padecen, los mismos que serán emprendidos o no en función de una gran diversidad de factores dentro de los cuales las cogniciones juegan un papel relevante. En particular las creencias sobre el control de la salud son vistas como factores más susceptibles de cambio o de influencia para lograr la modificación de conductas de cuidado de la salud, en comparación con otros elementos del comportamiento humano (Walker, 2001).

En general, las personas creen que algunos aspectos de su vida están controlados por factores internos (personales) o por una diversidad de factores externos (la suerte, el destino, o el poder de otras personas), los cuales impedirían o promoverían ciertos resultados o desenlaces. Esta dinámica hace referencia al locus de control (LC), que se deriva de la Teoría del Aprendizaje Social (TAS) establecida por Rotter (1954). La TAS sostiene que las creencias de control sobre aspectos de la vida o condiciones específicas pueden variar en una misma persona, denotando que la predicción del comportamiento podría estimarse de mejor manera con el desarrollo de instrumentos de medición específicos para tales aspectos o condiciones (Rotter, 1975), entre ellas la salud.

El desarrollo de investigación empírica sobre el LC en salud (LCS) llevó a diversos autores a aportar una nueva visión del LCS que coincide con los planteamientos de Rotter $(1954,1975)$ respecto del locus de control interno (LC-I), que hace referencia a que el individuo se cree directamente implicado en la producción de ciertos resultados y por lo tanto el reforzamiento recae en sí mismo. No obstante, esta perspectiva difiere de la teoría clásica al señalar que existe más de una dimensión del locus de control externo (LC-E), puesto que asume que los comportamientos de las personas pueden diferenciarse de manera clara cuando estas consideran que los resultados o acontecimientos en su vida son controlados por el azar (LCE-A), el poder de otros (LCE-OP) o el poder de Dios (LCE-Dios) (Wallston et al., 1999; Welton, Adkins, Ingle, \& Dixon, 1996).

Este aporte teórico dio paso al desarrollo de instrumentos para la estimación de las dimensiones propuestas. De esta manera un grupo de investigadores diseñaron la serie de escalas denominadas Multidimensional Health Locus of Control (MHLC), compuestas por las formas A, B y $\mathrm{C}$, que permiten analizar las múltiples dimensiones del locus de control en salud en adolescentes, jóvenes y adultos sanos o enfermos. Desde entonces esta familia de escalas ha sido ampliamente utilizada y adaptada a una diversidad de poblaciones con diferentes condiciones culturales y de salud (Wallston, 2005). Particularmente, la forma $C$ fue mejorada por Wallston (Wallston et al., 1999) con la adición de la escala conocida por su denominación en inglés como God Locus Of Health Control (GLHC).

El empleo de estas escalas ha permitido acumular evidencia a lo largo de casi cuatro décadas que apunta a que las personas que se sienten más en control de sí mismas (LCI) tienden a exhibir conductas más 
saludables (Wallston, 2005; Berglund, Lytsy, \& Westerling, 2014), lo que para el caso de la DM2 se interpreta como una mejor adherencia al tratamiento al producir un mejor control glucémico (Indelicato et al., 2017; Williams, Lymch, Voronca, \& Egede, 2016); en cambio, el LCE-A ha sido considerado como un predictor de mal control glucémico (Aberle et al., 2011; Morowatisharifabad, Mahmoodabad, Baghianimoghadam, \& Tonekaboni, 2010) y se ha asociado a mayores niveles de ansiedad estado en mujeres con diabetes gestacional (Miazgowski, Bikowska, Ogonowski, \& Taszarek, 2017). Por su parte, la influencia de Dios ha mostrado mejorar o empeorar los comportamientos en salud en poblaciones y condiciones diversas (Moore, 2014; Pomeranz et al., en prensa; Tsimtsiou, Kirana, \& Hatzichristou, 2014) y puede actuar como barrera o motivador de la adherencia al tratamiento (Ahmedani, Peterson, Wells, Rand, \& Williams, 2013; Kremer, Ironson, \& Porr, 2009) o de condiciones anómalas como la depresión en adultos mayores con enfermedades crónicas (Aflakseir \& MohammadAbadi, 2017).

Las creencias de control de Dios en la salud podrían tener implicaciones psicológicas benignas, por lo que sería de utilidad ampliar el estudio de estas influencias para impulsar un mejor acompañamiento en enfermos crónicos. Esto último tiene especial importancia en el contexto cultural mexicano donde aproximadamente el $95.1 \%$ de su población mayor de dieciocho años de edad practica alguna religión (Red de Investigadores del Fenómeno Religioso en México, 2016), lo cual ocurre independientemente de las diferencias de sexo, pues aunque se ha identificado un mayor porcentaje de mujeres que declaran profesar alguna religión, este porcentaje es muy cercano al reportado por hombres (Instituto Nacional de Estadística y Geografía, 2011). Adicionalmente se sabe que el $96 \%$ de los adultos de cincuenta años y más considera a la religión como un elemento muy importante o algo de importancia en su vida diaria (Benjamins, 2007).
A pesar de lo anterior, la influencia de la religiosidad sobre la salud ha sido pobremente estudiada en población mexicana (Rivera-Ledesma, López-Lena, \& Jiménez-Zavala, 2014) y específicamente, la información de la relación de las creencias de control de Dios sobre la DM2, no es concluyente. Sin embargo, hay indicios acerca de que su estudio podría ser relevante, ya que en población mexicana no diabética se ha encontrado que personas de cincuenta años o más que acuden a servicios religiosos al menos una vez a la semana tienen mayor probabilidad de realizarse pruebas de detección de diabetes y de colesterol. Asimismo, adultos mexicanos que consideran a la religión como algo muy importante en sus vidas son más propensos a realizarse otras pruebas de detección de otras enfermedades (Benjamins, 2007). Adicionalmente se ha identificado que en mexicanos que viven con diabetes y son religiosos prestan mejor cuidado y control de sus síntomas y esto se manifiesta de igual forma en hombres que en mujeres (Rivera-Hernandez, 2016).

Aún con los antecedentes previamente presentados y a pesar de la disponibilidad de la escala GLHC, su uso en población mexicana no se conoce y por lo tanto la contribución del constructo para la predicción del comportamiento en salud es aún inexplorada. En este sentido, el estudio psicométrico de la Escala de Locus de Control de la Salud en Dios (GLHC; Wallston et al., 1999) es pertinente en México, un país en donde la diabetes ha alcanzado niveles epidémicos y en el que no se dispone de un instrumento que permita determinar la influencia de creencias de control de Dios sobre las conductas deseables como la adherencia al tratamiento de la enfermedad, a pesar de la evidencia existente sobre la asociación entre el LCS de diversa índole y la adherencia al tratamiento.

Por ello, el presente trabajo tiene como objetivo determinar las propiedades psicométricas de la escala GLHC, con el propósito de aportar elementos que permitan una mejor comprensión de la conducta relacionada con la salud y el cumplimiento de los 
pacientes en el tratamiento de su enfermedad en el primer nivel de atención.

\section{Método}

\section{Participantes}

Se realizó un estudio instrumental (Ato, López, \& Benavente, 2013) en el que participaron 539 adultos mexicanos. La muestra del estudio estuvo integrada en su mayoría por mujeres (67.7\%), la edad de los participantes osciló entre 24 y 89 años $\left(M_{\text {edad }}=61.8\right.$; $D E=10.9$ ). Del total, $53.8 \%$ fueron originarios de alguna zona rural aunque en el momento de la encuesta el 90.5\% refirió residir en una zona urbana; $41.6 \%$ realizan alguna actividad por la que reciben alguna remuneración económica, siendo la mediana del ingreso familiar mensual de $\$ 2,450.00$ (1800.005000.00 pesos mexicanos), equivalente a 1.2 salarios mínimos diarios, lo que da cuenta del bajo nivel socioeconómico del grupo de estudio. La media de duración de la DM2 fue de 11.7 años $(D E=3.1)$ otras características demográficas y clínicas del grupo de estudio se presentan en la Tabla 1.

Los participantes del estudio fueron elegidos por el método de muestreo aleatorio simple a partir del censo nominal de pacientes con DM2. Posteriormente fueron localizados cuando acudieron a la unidad médica a su cita de control más próxima. Cada paciente elegido fue invitado a participar informándole de manera oral y por escrito el objetivo de la investigación, así como de su derecho de colaborar voluntariamente y de poder retirarse en cualquier momento si era su deseo. Quienes aceptaron la invitación firmaron un consentimiento informado. Cuando alguien se negó a participar se generó un nuevo número aleatorio para invitar a otro paciente. Los criterios de inclusión fueron: ser usuario con vigencia de derechos de seguridad social de una unidad de primer nivel de atención en Xalapa, Veracruz, México, con diagnóstico confirmado de DM2, tener una edad igual o mayor a veinte años y no padecer hipoacusia o alguna condición que impidiera responder con coherencia el interrogatorio. El periodo de inclusión ocurrió del 4 de enero y el 31 de mayo del 2016.

Tabla 1

Características demográficas y clínicas de los participantes

\begin{tabular}{|c|c|c|}
\hline & $n$ & $\%$ \\
\hline \multicolumn{3}{|l|}{ Escolaridad } \\
\hline Analfabeta & 38 & 7.1 \\
\hline Sabe leer y escribir & 120 & 22.3 \\
\hline Educación básica completa & 252 & 46.7 \\
\hline Educación media completa & 56 & 10.4 \\
\hline Educación superior & 73 & 13.5 \\
\hline \multicolumn{3}{|l|}{ Estado civil } \\
\hline Casado & 284 & 52.7 \\
\hline Unión libre & 67 & 12.4 \\
\hline Soltero & 78 & 14.5 \\
\hline Viudo & 90 & 16.7 \\
\hline Divorciado & 20 & 3.7 \\
\hline \multicolumn{3}{|l|}{ Ocupación } \\
\hline Empleado & 177 & 32.9 \\
\hline Desempleado & 11 & 2.0 \\
\hline Autoempleado & 47 & 8.7 \\
\hline Jubilado & 96 & 17.8 \\
\hline Ama de casa & 208 & 38.6 \\
\hline \multicolumn{3}{|l|}{ Tipo de tratamiento } \\
\hline Hipoglucemiantes orales & 416 & 77.2 \\
\hline Insulina & 17 & 3.2 \\
\hline \multicolumn{3}{|l|}{ Hipoglucemiantes orales } \\
\hline e insulina & 103 & 19.1 \\
\hline Dieta y ejercicio & 3 & 6 \\
\hline \multicolumn{3}{|c|}{ Acude a control mensual de la diabetes } \\
\hline Sí & 513 & 95.2 \\
\hline No & 26 & 4.8 \\
\hline \multicolumn{3}{|l|}{$\begin{array}{l}\text { Tiene diagnóstico de otra } \\
\text { enfermedad crónica }\end{array}$} \\
\hline Sí & 350 & 64.9 \\
\hline No & 189 & 35.1 \\
\hline
\end{tabular}

Nota: $n=$ número; $\%=$ porcentaje 


\section{Instrumentos}

Cuestionario de datos demográficos y clínicos. Se diseñó un cuestionario para recopilar los datos demográficos y clínicos de los pacientes a través del cual se indagaron: sexo, edad, estado civil, nivel de educación, ingresos familiares, lugar de residencia, años de duración de la diabetes, tipo de tratamiento para la diabetes, comorbilidad y frecuencia de asistencia a citas para el tratamiento de la diabetes.

Escala de Locus de Control de la Salud en Dios (GLHC; Wallston et al., 1999). La escala GLHC está constituida por seis afirmaciones con opciones de respuesta en escala de tipo Likert de seis puntos que van desde totalmente en desacuerdo con valor de un 1 , hasta totalmente de acuerdo con valor de 6 , por lo que la puntuación total de la escala oscila de seis a 36 puntos posibles. La consistencia interna de la escala fue estimada a través del coeficiente de alpha en una muestra de pacientes con artritis reumatoide y otra más de pacientes con esclerosis sistémica, habiendo obtenido coeficientes aceptables de .87 y de .94 , respectivamente.

\section{Procedimiento}

\section{Adaptación transcultural de la escala de GLHC}

Inicialmente se procedió a realizar la adaptación transcultural de la escala de GLHC siguiendo los lineamientos de las guías acreditadas por RamadaRodilla, Serra-Pujadas y Delclós-Clanchet (2013). Primero fueron traducidas por un experto en lenguaje clínico, después fueron sometidas al criterio de un panel de tres jueces con experiencia en la teoría del locus de control en salud, a quienes se les pidió evaluar la congruencia teórica de los ítems. Posteriormente, otro panel de tres jueces evaluó la claridad de la redacción y la pertinencia cultural del lenguaje. A cada nuevo ítem satisfactoriamente traducido se le asignaron tres puntos, los ítems puntuados por debajo de tres fueron modificados nuevamente. La revisión por los paneles de jueces implicó dos rondas, que concluyeron cuando todos estuvieron de acuerdo con las modificaciones realizadas y la equivalencia semántica de los términos traducidos.

Posterior a la adaptación transcultural se efectuó una prueba piloto, con la intención de identificar la adecuada comprensión de las afirmaciones. En ella participaron 60 pacientes con diagnóstico de DM2 elegidos por muestreo a conveniencia en todos los turnos de una unidad de primer nivel de atención. La edad promedio fue de 59 años $(D E=6.5)$, en su mayoría mujeres (68.3\%) y viviendo en pareja (81.6\%). El 53.3\% realizaba alguna actividad por la que reciben remuneración económica, 15\% no sabía leer ni escribir, 6.6\% sabían leer y escribir pero no terminaron sus estudios de primaria, mientras que el 31\% únicamente terminó la educación primaria. Tras la prueba piloto no hubo problemas de comprensión de los ítems por lo que la aplicación se realizó con la versión de la escala previamente aprobada por los jueces, sin embargo, dada la baja escolaridad de casi la tercera parte de los participantes se optó por aplicar las escalas en formato de entrevista.

\section{Análisis de datos}

Análisis descriptivo. Fue realizado un análisis de medidas de tendencia central y distribucional de los ítems.

Invarianza de medición y comparación de medias latentes. Se realizó un análisis factorial de grupo múltiple con el programa EQS 6.2 (Bentler \& Wu, 2012), usando el método de máxima verosimilitud robusto (MVR) ya que por sus características distribucionales, los ítems poseen algún grado de nonormalidad (Micceri, 1989). El ajuste de los modelos fue valorado con diversos índices (CFI, RMSEA, SRMR, y la prueba general $\chi^{2}$ con la corrección Satorra-Bentler). La $S B-\chi^{2}$ (Satorra \& Bentler, 1994) tiende a estar influida por el tamaño muestral por lo que su interpretación debe realizarse con cautela. 
Fueron analizados diferentes grados de invarianza de medición considerando el sexo (varones y mujeres) y la edad (menor que 60 años, y 60 años o más). Para comenzar, fue evaluada la igualdad de la configuración del instrumento entre los grupos (invarianza configuracional), y posteriormente la equivalencia de las cargas factoriales (invarianza métrica), de los interceptos (invarianza fuerte) y de los residuales entre los grupos (invarianza estricta) (Byrne, 2008), siendo el cumplimiento de invarianza fuerte el requisito necesario para comparar medias (latentes y observadas). Es necesario precisar que los interceptos de los ítems fueron especificados desde el inicio, y no desde la evaluación de la invarianza fuerte, a fin de lograr una correcta comparación de modelos anidados (Dominguez-Lara, Fernández-Arata, Manrique, Alarcón-Parco, \& DíazPeñaloza, en prensa).

Fueron considerados dos criterios para la valoración de los diferentes grados de invarianza, uno global y otro específico. El primero consideró la variación en los índices de ajuste, es decir, el cambio en el CFI $\left(\mathrm{CFI}_{1}-\mathrm{CFI}_{2}<.01\right.$; Cheung \& Rensvold, 2002), el RMSEA (RMSEA - RMSEA $_{1}<.015$; Chen, 2007), y SRMR $\left(\mathrm{SRMR}_{2}-\mathrm{SRMR}_{1}<.030\right.$; Chen, 2007) entre modelos anidados. Por ejemplo, se espera que el CFI del modelo más restricto $\left(\mathrm{CFI}_{2}\right)$, difiera del $\mathrm{CFI}_{1}$ (modelo menos restricto) como máximo en .01. El criterio más específico se enfocó en el análisis individual de la significancia estadística de los índices de modificación (IM; Sörbom, 1989) asociados a cada restricción. De este modo, si el $\chi^{2}$ es estadísticamente significativo $(p<.05)$, se sugiere relajar la restricción, es decir, que los ítems no son estadísticamente equivalentes en el grado de invarianza evaluado (sean cargas factoriales, interceptos, o residuales). En este sentido, un $\chi^{2}$ estadísticamente significativo $(p<.05)$ asociado a cada restricción da evidencia desfavorable para el grado de invarianza que se esté evaluando.

Sobre la base del cumplimiento de la invarianza fuerte, fueron comparadas las medias latentes entre grupos manteniendo la igualdad de interceptos, y especificando la media de uno de los grupos a cero y dejando libre la media del otro grupo (Brown, 2006).

Análisis dimensional. Luego de realizar el análisis de invarianza, fue evaluado el modelo unidimensional fue evaluado con la muestra completa con el objetivo de obtener la versión final del instrumento. Complementariamente, se evaluó la validez interna convergente mediante el cálculo de la Varianza Extraída Promedio (AVE; Fornell \& Larcker, 1981), cuyos valores por encima de .50 se consideran aceptables.

Confiabilidad. Basados en el modelo congenérico, fue evaluado el modelo tau-equivalente, el cual asume igualdad estadística de cargas factoriales (Dunn, Baguley, \& Brunsden, 2014) fue evaluado a fin de justificar el uso del coeficiente á (Cronbach, 1951) para estimar la confiabilidad de las puntuaciones. Para determinar la aproximación a dicho modelo de medición, fueron usados los criterios basados en las variaciones en el CFI, el RMSEA y SRMR descritos anteriormente. En vista de los resultados, fue evaluada la confiabilidad de la variable latente se evaluó mediante dos coeficientes: el $\omega$ (McDonald, 1999) у $H$ (Dominguez-Lara, 2016a; Hancock \& Mueller, 2001); y la confiabilidad de las puntuaciones mediante el coeficiente $\alpha$ con intervalos de confianza (IC; Dominguez-Lara \& Merino-Soto, 2015).

De forma complementaria fue analizado el impacto de los residuales correlacionados sobre los indicadores de confiabilidad obtenidos debido a que es probable que exista algún grado de varianza irrelevante al constructo producto de la similitud en el fraseo de varios ítems. Para tal fin, fue usada una fórmula de corrección para el coeficiente $\alpha$ (Dominguez-Lara \& Merino-Soto, 2017) y coeficiente $\omega$ (Dominguez-Lara, 2016b; Raykov, 2001). 
Tabla 2

Análisis descriptivo de los ítems por sexo y edad

\begin{tabular}{|c|c|c|c|c|c|c|c|c|c|c|c|c|c|c|c|c|}
\hline & \multicolumn{8}{|c|}{ Sexo } & \multicolumn{8}{|c|}{ Edad } \\
\hline & \multicolumn{4}{|c|}{ Varones } & \multicolumn{4}{|c|}{ Mujeres } & \multicolumn{4}{|c|}{ Grupo 1} & \multicolumn{4}{|c|}{ Grupo 2} \\
\hline Ítem & $M$ & $D E$ & $g_{1}$ & $g_{2}$ & $M$ & $D E$ & $g_{1}$ & $g_{2}$ & $M$ & $D E$ & $g_{1}$ & $g_{2}$ & $M$ & $D E$ & $g_{1}$ & $g_{2}$ \\
\hline 1 & 3.870 & 2.202 & -.327 & -1.718 & 3.900 & 2.221 & -.360 & -1.697 & 3.380 & 2.262 & .071 & -1.85 & 4.253 & 2.106 & -.669 & -1.328 \\
\hline 2 & 2.770 & 2.094 & .584 & -1.424 & 2.550 & 2.089 & .826 & -1.129 & 2.190 & 1.902 & 1.204 & -.307 & 2.918 & 2.169 & .477 & -1.574 \\
\hline 3 & 3.070 & 2.157 & 319 & -1.708 & 2.720 & 2.177 & .627 & -1.447 & 2.570 & 2.114 & .774 & -1.224 & 3.016 & 2.202 & .361 & -1.699 \\
\hline 4 & 2.870 & 2.184 & .490 & -1.594 & 2.780 & 2.164 & .569 & -1.498 & 2.360 & 2.013 & 1.007 & -.775 & 3.130 & 2.221 & .262 & -1.758 \\
\hline 5 & 3.070 & 2.197 & 288 & -1.753 & 3.020 & 2.234 & .352 & -1.724 & 2.650 & 2.159 & .693 & -1.375 & 3.313 & 2.225 & .098 & -1.816 \\
\hline 6 & 2.930 & 2.165 & .394 & -1.670 & 2.720 & 2.123 & .611 & -1.424 & 2.340 & 1.991 & 1.010 & -.744 & 3.101 & 2.183 & .255 & -1.745 \\
\hline
\end{tabular}

Nota: $M=$ Media; $D E=$ Desviación estándar; $g_{1}=$ Asimetría; $g_{2}=$ Curtosis; Grupo $1=$ Menores de 60 años; Grupo $2=60$ años o más

\section{Resultados}

Estadísticos descriptivos. Los ítems en su mayoría se inclinan hacia el polo más bajo de la distribución (Tabla 2). Asimismo, los indicadores de curtosis están cerca de los límites considerados cercanos a la normalidad univariada (+/- 1.5; Pérez \& Medrano, 2010), y la asimetría es adecuada en la mayoría de los casos $(<|1.000|)$.

Invarianza de medición y comparación de medias latentes. El análisis factorial realizado de forma independiente en cada uno de los grupos (sexo y edad) indica que todos presentan índices de ajuste satisfactorios (Tabla 3). Con relación al sexo, el ajuste de la invarianza configural es adecuado, y una vez restringida la igualdad de cargas factoriales, el cambio mínimo en los índices de ajuste (CFI, RMSEA, SRMR) y la presencia de IM estadísticamente no significativos indica que se cumple satisfactoriamente la invarianza métrica. Un panorama similar ocurrió cuando se realizó la restricción de la igualdad de interceptos sobre la base del modelo anterior, derivando en el cumpliendo así la invarianza fuerte. Por último, la invarianza estricta, referida a la igualdad estadística de residuales, también obtuvo evidencia favorable.
Con respecto a la edad, la invarianza configural y métrica obtuvieron evidencia favorable (Tabla 3), tanto con relación a los índices de ajuste como a los IM. Sin embargo, al evaluar la invarianza fuerte, pese a que la variación en los índices de ajuste brinda evidencia favorable, el análisis individual de los IM indica que el intercepto de los ítems uno $\left(\chi^{2}=6.316\right.$, $p=.012)$ y tres $\left(\chi^{2}=9.182, p=.002\right)$ no son invariantes entre los grupos. En este caso, se procedió a relajar dichas restricciones, corroborando su impacto en el ajuste de modelo. Por tal motivo, en cuanto a la edad, solo se logró una invarianza fuerte parcial.

Una vez obtenida evidencia a favor de la invarianza fuerte, se procedió a comparar las medias latentes entre varones y mujeres manteniendo la igualdad de interceptos, y especificando la media de uno de los grupos a cero (en este caso varones) y la media del otro grupo, libre. En este caso, la diferencia de medias no fue estadísticamente significativa (diferencia $=-.098, p>.05$ ). 
Tabla 3

Índices de ajuste y evaluación de la invarianza de medición

\begin{tabular}{|c|c|c|c|c|c|c|}
\hline & CFI & $\begin{array}{l}\text { RMSEA } \\
\text { (IC90\%) }\end{array}$ & SRMR & $\begin{array}{c}\Delta \text { CFI } \\
\text { (IC90\%) }\end{array}$ & $\triangle \mathrm{RMSEA}$ & $\Delta \mathrm{SRMR}$ \\
\hline \multicolumn{7}{|l|}{ Sexo } \\
\hline Varones & .999 & $\begin{array}{c}.024 \\
(.000, .091)\end{array}$ & .027 & & & \\
\hline Mujeres & 1.000 & $\begin{array}{c}.000 \\
(.000, .053)\end{array}$ & .018 & & & \\
\hline Configural & 1.000 & $\begin{array}{c}.015 \\
(.000, .057)\end{array}$ & .023 & & & \\
\hline Métrica & .999 & $\begin{array}{c}.014 \\
(.000, .052)\end{array}$ & .039 & -.001 & -.001 & .016 \\
\hline Fuerte & .998 & $\begin{array}{c}.030 \\
(.000, .061)\end{array}$ & .039 & -.001 & .016 & .000 \\
\hline Estricta & .998 & $\begin{array}{c}.025 \\
(.000, .055)\end{array}$ & .036 & .000 & -.005 & -.003 \\
\hline \multicolumn{7}{|c|}{ Grupo de edad } \\
\hline$\leq 59$ años & .996 & $\begin{array}{c}.041 \\
(.000, .091)\end{array}$ & .026 & & & \\
\hline$\leq 60$ años & .996 & $\begin{array}{c}.048 \\
(.005, .095)\end{array}$ & .026 & & & \\
\hline Configural & .995 & $\begin{array}{c}.049 \\
(.008, .079)\end{array}$ & .026 & & & \\
\hline Métrica & .992 & $\begin{array}{c}.053 \\
(.025, .079)\end{array}$ & .063 & -.003 & .004 & .037 \\
\hline Fuerte & .989 & $\begin{array}{c}.064 \\
(.039, .088)\end{array}$ & .063 & -.003 & .011 & .000 \\
\hline Fuerte $_{\text {parcial }}$ & .992 & $\begin{array}{c}.055 \\
(.026, .081)\end{array}$ & .054 & .000 & .002 & -.009 \\
\hline
\end{tabular}


Análisis dimensional y confiabilidad. Una vez corroborada la invarianza de medición con relación al sexo, fue analizada la muestra completa y el modelo unidimensional (congenérico) obtuvo índices de ajuste satisfactorios $\left(\mathrm{SB}-\chi^{2}{ }_{(9)}=13.878, p=.127\right.$; CFI $=.998 ;$ RMSEA $=.032$ [IC90\% .000, .063], SRMR $=.017)$, cargas factoriales entre moderadas $\mathrm{y}$ altas $\left(\lambda_{\text {promedio }}=.782\right) \mathrm{y}$ adecuada validez interna convergente $(\mathrm{AVE}=.621)$.

Con el objetivo de justificar el uso del coeficiente $\alpha$ fue restringida la igualdad de cargas factoriales (modelo tau equivalente) obteniendo índices de ajuste satisfactorios $\left(\mathrm{SB}-\chi_{(14)}^{2}=54.724, p<.001\right.$; CFI $=$ .984; RMSEA = .074 [IC90\% .054, .094], SRMR = .118) y cuya diferencia respecto al modelo congenérico parece no ser relevante. No obstante, un análisis individual de los IM asociados a cada restricción indica que en su mayoría fueron estadísticamente significativos $(p<.05)$, por lo que no es posible asumir la tau-equivalencia de los ítems. Con todo, el cálculo del coeficiente $\alpha$ indicó una magnitud cerca al coeficiente $\omega$, lo que indicaría que pese a no cumplir con la igualdad de cargas factoriales, la infraestimación no es relevante (Tabla 4).

Luego de ello, fue estimada la confiabilidad del constructo utilizando los coeficientes $\omega$ y $H$ (Tabla 4), cuyo único requerimiento es que los ítems sean medidas congenéricas, encontrando en ambos casos magnitudes elevadas (> .90). Adicionalmente, los IM indicaron la necesidad de correlacionar tres pares de residuales a fin de mejorar el ajuste del modelo, aunque un uso más preciso de esa información indicaría en qué grado la confiabilidad del constructo está infra o sobreestimado (Raykov, 2001). De este modo, una vez realizado el cálculo de las correlaciones entre residuales $\left(\varphi_{2,5}=-.077 ; \varphi_{2,3}=\right.$ $\left..161 ; \varphi_{1,3}=-.122\right)$, los coeficientes $\alpha_{\text {corregido }} \mathrm{y}$ $\omega_{\text {corregido }}$ obtuvieron magnitudes superiores, aunque no en gran medida, en comparación a las presentadas previamente (Tabla 4).
Tabla 4

Cargas factoriales y confiabilidad (muestra total)

\begin{tabular}{lc}
\hline Ítems & $\lambda$ \\
\hline 1 & .572 \\
2 & .763 \\
3 & .850 \\
4 & .846 \\
5 & .838 \\
6 & .823 \\
& $.903(.886, .917)$ \\
$\alpha($ IC95\%) & .915 \\
$\alpha_{\text {corregido }}$ & .906 \\
$\omega$ & .909 \\
$\omega_{\text {corregido }}$ & .920 \\
$H$
\end{tabular}

Nota: $\lambda=$ cargas factoriales; $\alpha_{\text {corregido }} \mathrm{y} \omega_{\text {corregido }}$ : coeficientes corregidos ante la presencia de residuales correlacionados.

\section{Discusión}

En nuestro conocimiento, este es el primer trabajo publicado en español donde se reportan las evidencias de validez de la GLHC en personas con DM2 en el contexto de una región de México. La GLHC mostró una solución factorial teóricamente concordante con la escala original e indicadores de confiabilidad robustos, tanto de puntajes observados como de variables latentes, por lo que puede ser considerada como una escala con una sólida estructura interna e fuertes indicadores de confiabilidad para su aplicación en población mexicana, con utilidad relevante para la exploración de las creencias de control en salud que permita contribuir a la comprensión de los comportamientos en salud de la población de interés.

Un aspecto relevante es el análisis equidad de medida (invarianza de medición) con relación al sexo del participante, ya que no fueron halladas diferencias significativas entre varones y mujeres, lo que posiblemente se dé porque no han sido reportadas 
diferencias en cuanto a la religiosidad por sexo en la población mexicana con los datos hasta el momento conocidos, lo que fue corroborado con los resultados. No obstante, con respecto a la edad, existen algunos ítems que estarían sesgados hacia el grupo de mayor edad, es decir, que las diferencias observadas podrían atribuirse a otras factores y no al constructo evaluado (LC-Dios), es posible que pueda explicarse por un incremento de la fe o la religiosidad conforme la edad aumenta en los habitantes de la región en la que se situó esta investigación, quienes al sentirse vulnerados por las enfermedades crónicas, la disminución natural de la fuerza física o bien la discapacidad o cambios en su rol social, incrementan sus creencias en Dios (Palacios, 2015), por lo que no es posible usar el GLHC para realizar un análisis comparativo respecto a la edad.

Finalmente, a pesar de la robustez mostrada por los indicadores de confiabilidad y validez, es necesario reconocer que la falta de exploración sobre la afiliación religiosa y la falta de medidas objetivas de religiosidad limitaron la caracterización de la muestra de participantes, por ello para ampliar la evidencia de validez de este constructo en población mexicana se recomienda que en el futuro se considere el análisis por subgrupos de religiones y de niveles de religiosidad. Otro aspecto importante a considerar en la acumulación de la evidencia de validez de la escala, es que el alcance de resultados de la presente investigación se restringe a una interpretación local, por lo es recomendable que en futuras investigaciones se amplíe la muestra de estudio con la finalidad de verificar la validez de la escala en el contexto nacional, así como su relación con indicadores biológicos en estudios longitudinales para que de ese modo pueda apreciarse su impacto en el curso de la enfermedad.

Incluso en presencia de las limitaciones identificadas en el desarrollo del presente trabajo, los resultados presentados pueden considerarse un aporte importante para la comprensión del LCS específico para DM2 en población mexicana, su fácil aplicación e interpretación hacen posible su utilización en unidades de primer nivel de atención, con lo que se da el primer paso para identificar el verdadero valor del locus de control en Dios como predictor de situaciones clínicas tan relevantes como la adherencia al tratamiento en pacientes con esta condición en el contexto mexicano. 


\section{Referencias}

Aberle, I., Scholz, U., Bach-Kliegel, B., Fischer, C., Gorny, M., Langer, K., \& Kliegel, M. (2009). Psychological aspects in continuous subcutaneous insulin infusion: a retrospective study. Journal of Psychology, 143(2), 147-160. doi: 10.3200/JRLP.143.2.147-160

Aflakseir, A.-A., \& Mohammad-Abadi, M.-S. (2016). The role of health locus of control in predicting depression symptoms in a sample of Iranian older adults with chronic diseases. Iranian Journal of Psychiatry, 11(2), 82-86.

Ahmedani, B. K., Peterson, E. L., Wells, K. E., Rand, C. S., \& Williams, L. K. (2013). Asthma medication adherence: the role of god and other health locus of control factors. Annals of Allergy, Asthma \& Immunology, 110(2), 75-79. doi: 10.1016/j.anai.2012.11.006

Ato, M., López, J., \& Benavente, A. (2013). Un sistema de clasificación de los diseños de investigación en psicología. Anales de Psicología, 29(3), 1038-1059.

Benjamins, M. R. (2007). Predictors of preventive health care use among middle-aged and older adults in Mexico: The role of religion. Journal of Cross-Cultural Gerontology, 22(2), 221-234. doi: 10.1007/s10823-0079036-4

Bentler, P. M., \& Wu, E. J. C. (2012). EQS 6.2 for Windows [Statistical Program]. Encino, Estados Unidos: Multivariate Software, Inc.

Berglund, E., Lytsy, P., \& Westerling, R. (2014). The influence of locus of control on self-rated health in context of chronic disease: a structural equation modeling approach in a cross sectional study. BMC Public Health, 14(1), 492. doi: 10.1186/1471-2458-14-492

Brown, T. (2006). Confirmatory Factor Analysis for Applied Research. Nueva York, Estados Unidos: The Guilford Press.

Byrne, B. (2008). Testing for multigroup equivalence of a measuring instrument: A walk through the process. Psicothema, 20, 872-882.

Chaplin, W. F., Sparrow, V., Stuhr, J., \& Van, E. (2001). A Structural Evaluation of the Expanded Multidimensional Health Locus of Control Scale with a Diverse Sample of Caucasian / European, Native, and Black Canadian Women. Journal of Health Psychology, 6(4), 447-455.
Chen, F. F. (2007). Sensitivity of goodness of fit indexes to lack of measurement invariance. Structural Equation Modeling, 14, 464-504. doi: 10.1080/10705510701301834

Cheung, G. W., \& Rensvold, R. B. (2002). Evaluating goodness-of-fit indexes for testing measurement invariance. Structural Equation Modeling, 9(2), 233255. doi: 10.1207/S15328007SEM0902_5

Cronbach L. J. (1951). Coefficient alpha and the internal structure of tests. Psychometrika, 16(3), 297-334. doi: 10.1007/BF02310555

Dominguez-Lara, S. (2016a). Evaluación de la confiabilidad del constructo mediante el Coeficiente $\mathrm{H}$ : Breve revisión conceptual y aplicaciones. Psychologia: Avances en la disciplina, 10(2), 87-94. doi: 10.21500/ 19002386.2134

Dominguez-Lara, S. (2016b). Errores correlacionados y estimación de la fiabilidad en estudios de validación: Comentarios al trabajo validación de la escala ehealth literacy (eheals) en población universitaria española. Revista Española de Salud Pública, 90(9), e1-e2.

Dominguez-Lara, S., \& Merino-Soto, C. (2015). ¿Por qué es importante reportar los intervalos de confianza del coeficiente alfa de Cronbach? Revista Latinoamericana de Ciencias Sociales, Niñez y Juventud, 13(2), 1326-1328.

Dominguez-Lara, S., \& Merino-Soto, C. (2017). Una modificación del coeficiente alfa de Cronbach por errores correlacionados. Revista Médica de Chile, 145(2), 270-271.

Dominguez-Lara, S., Fernández-Arata, M., Manrique, D., Alarcón-Parco, D., \& Díaz-Peñaloza, M. (en prensa). Datos normativos de una escala de agotamiento emocional académico en estudiantes universitarios de psicología de Lima (Perú). Educación Médica. doi: 10.1016/j.edumed.2017.09.002

Dunn, T. J., Baguley, T., \& Brunsden, V. (2014). From alpha to omega: A practical solution to the pervasive problem of internal consistency estimation. British Journal of Psychology, 105(3), 399-412. doi: 10.1111/bjop.12046

Edelman, S. V., \& Polonsky, W. H. (2017). Type 2 diabetes in the real world: The elusive nature of glycemic control. Diabetes Care. doi: 10.2337/dc16-1974 
Fornell, C., \& Larcker, D. F. (1981). Evaluating structural equation models with unobservable variables and measurement error. Journal of Marketing Research, 18, 39-50.

Hancock, G. R., \& Mueller, R. O. (2001). Rethinking construct reliability within latent variable systems. En R. Cudeck, S. H. C. du Toit \& D. Sörbom (Eds.), Structural equation modeling: Past and present. A Festschrift in honor of Karl G. Jöreskog (pp. 195-261). Chicago, Estados Unidos: Scientific Software International.

Hernández-Ávila, M., Gutiérrez, J. P., \& Reynoso-Noverón, N. (2013). Diabetes mellitus en México. El estado de la epidemia. Salud Pública de México, 55(1), 129-136. Recuperado de http://www.scielo.org.mx/pdf/spm/ v55s2/v55s2a10.pdf

Indelicato, L., Mariano, V., Galasso, S., Boscari, F., Cipponeri, E., Negri, C., ... Bruttomesso, D. (2017). Influence of health locus of control and fear of hypoglycaemia on glycaemic control and treatment satisfaction in people with Type 1 diabetes on insulin pump therapy. Diabetic Medicine, 34(5), 691-697. doi: 10.1111/dme.13321

Instituto Nacional de Estadística y Geografía. (2011). Panorama de las religiones en México 2010. México: Instituto Nacional de Estadística y Geografía, Secretaría de Gobernación. Recuperado de http://internet. contenidos.inegi.org.mx/contenidos/Productos/ prod_serv/contenidos/espanol/bvinegi/productos/ censos/poblacion/2010/panora_religion/ religiones_2010.pdf

Jiménez-Corona, A., \& Aguilar-Salinas, C. A. (2013). Diabetes mellitus tipo 2 y frecuencia de acciones para su prevención y control. Salud Pública de México, 55(1), 137-143.

Kremer, H., Ironson, G., \& Porr, M. (2009). Spiritual and mind-body beliefs as barriers and motivators to HIVtreatment decision-making and medication adherence? A qualitative study. AIDS Patient Care and STDs, 23(2), 127-134. doi: 10.1089/apc.2008.0131

McDonald, R. P. (1999). Test theory: A unified treatment. Mahwah, Estados Unidos: L. Erlbaum Associates.

Miazgowski, T., Bikowska, M., Ogonowski, J., \& Taszarek, A. (2017). The Impact of Health Locus of Control and Anxiety on Self-Monitored Blood Glucose Concentration in Women with Gestational Diabetes Mellitus. Journal of women's health, O(0), 1-7. doi: 10.1089/jwh.2017.6366

Micceri, T. (1989). The unicorn, the normal curve, and other improbable creatures. Psychological Bulletin, 105, 156-166. doi: 10.1037/0033-2909.105.1.156

Morowatisharifabad, M. A., Mahmoodabad, S. S., Baghianimoghadam, M. H., \& Tonekaboni, N. R. (2010). Relationships between locus of control and adherence to diabetes regimen in a sample of Iranians. International Journal of Diabetes in Developing Countries, 30(1), 27-32. doi: 10.4103/0973-3930.60009

Moore, E. W. (2014). Assessing god locus of control as a factor in college students' alcohol use and sexual behavior. Journal of American College Health, 62(8), 578-587. doi: 10.1080/07448481.2014.947994

Palacios, F. R. V. (2015). La metamorfosis de la fe en creyentes de edad avanzada. Península, 10(1), 49-71. doi: 10.1016/j.pnsla.2014.08.001

Pérez, E., \& Medrano, L. (2010) Análisis Factorial Exploratorio: Bases Conceptuales y metodológicas. Revista Argentina de Ciencias del Comportamiento, 2(1), 58-66.

Pomeranz, M., Arbib, N., Haddif, L., Reissner, H., Romem, Y., \& Biron, T. (en prensa). «In God we trust» and other factors influencing trial of labor versus Repeat cesarean section. Journal of Maternal-Fetal and Neonatal Medicine. doi: 10.1080/14767058.2017.1326906

Ramada-Rodilla, J. M., Serra-Pujadas C., \& Delclós-Clanchet, G. L. (2013). Adaptación cultural y validación de cuestionarios de salud: Revisión y recomendaciones metodológicas. Salud Pública de México, 55(1), 57-66.

Raykov, T. (2001). Bias in coefficient alpha for fixed congeneric measures with correlated errors. Applied Psychological Measurement, 25(1), 69-76. doi: 10.1177/ 01466216010251005

Red de Investigadores del Fenómeno Religioso en México. (2016). Encuesta Nacional sobre Creencias y Prácticas Religiosas en México ENCREER/RIFREM 2016. México.

Rivera-Hernandez, M. (2016). Religiosity, social support and care associated with health in older Mexicans with diabetes. Journal of Religion and Health, 55(4), 165187. doi: $10.1007 / 128$ 
Rivera-Ledesma, A., López-Lena, M. M., \& Jiménez-Zavala, S. (2014). Espiritualidad, psicología y salud. Psicología y Salud, 24(55), 139-152.

Rotter, J. B. (1954). Social Learning a Clinical Psychology. Nueva York, Estados Unidos: Prentince Hall.

Rotter, J. B. (1975). Some problems and misconceptions related to the construct of internal versus external control of reinforcement. Journal of Consulting and Clinical Psychology, 43(1), 56-67.

Satorra, A., \& Bentler, P. M. (1994). Corrections to test statistics and standard errors in covariance structure analysis. En A. von Eye \& C. C. Clogg (Eds.), Latent variables analysis: Applications for developmental research (pp. 399-419). Thousand Oaks, Estados Unidos: Sage.

Secretaría de Salud. (2016). Encuesta Nacional de Salud y Nutrición de Medio Camino 2016. (ENSANUT MC 2016). Instituto Nacional de Salud Pública. doi: $10.21149 / 8593$

Shams, N., Amjad, S., Kumar, N., Ahmed, W., \& Saleem, F. (2016). Drug non-adherence in type 2 diabetes mellitus: Predictors and associations. Journal of Ayub Medical College Abbottabad-Pakistan, 28(2), 302-307.

Sörbom, D. (1989). Model modification. Psychometrika, 54(3), 371-384. doi: 10.1007/BF02294623
Tsimtsiou, Z., Kirana, P. S., \& Hatzichristou, D. (2014). Determinants of patients' attitudes toward patientcentered care: A cross-sectional study in Greece. Patient Education and Counseling, 97(3), 391-395. doi: 10.1016/j.pec.2014.08.007

Walker, J. M. (2001). Control and the psychology of health: theory, measurement and applications. Estados Unidos: Open University.

Wallston, K. A. (2005). The Validity of the Multidimensional Health Locus of Control Scales. Journal of Health Psychology, 10(5), 623-631. doi: 10.1177/1359105305055304

Wallston, K. A., Malcarne, V. L., Flores, L., Hansdottir, I., Smith, C. A., Stein, M. J., ... Clements, P. J. (1999). Does God determine your health? The God Locus of Health Control Scale. Cognitive Therapy and Research, 23(2), 131-142.

Welton, G. L., Adkins, A. G., Ingle, S. L., \& Dixon, W. A. (1996). God control: The fourth dimension. Journal of Psychology and Theology, 24(1), 13-25.

Williams, J. S., Lynch, C. P., Voronca, D., \& Egede, L. E. (2016). Health locus of control and cardiovascular risk factors in veterans with type 2 diabetes. Endocrine, 51(1), 83-90. doi: 10.1007/s12020-015-0677-8 


\section{Vianey Guadalupe Argüelles-Nava}

Instituto de Salud Pública, Universidad Veracruzana, México

Licenciada en Nutrición, Maestra en Salud Pública con área disciplinar en Epidemiología y Doctorante en Psicología por la Universidad Veracruzana. Coordinadora de la Maestría en Epidemiología, académica por tiempo determinado.

Autora corresponsal: vianeyarguellesn@gmail.com, varguelles@uv.mx

\section{Sergio Dominguez-Lara}

Instituto de Investigación de Psicología, Universidad de San Martín de Porres, Perú

Psicólogo y Magíster en Psicología. Docente del Instituto de Investigación de Psicología de la Facultad de Ciencias de la Comunicación, Turismo y Psicología de la Universidad de San Martín de Porres. Autor de artículos de corte metodológico y psicométrico en revistas arbitradas y con índice de impacto internacionales.

sdominguezl@usmp.pe

\section{Jaime Morales-Romero}

Instituto de Salud Pública, Universidad Veracruzana, México

Doctor en ciencias de la salud pública, médico especialista en epidemiología e investigador de tiempo completo adscrito al Instituto de Salud Pública de la Universidad Veracruzana. Miembro del Sistema Nacional de Investigadores del CONACYT (México). Coautor de artículos publicados en revistas nacionales e internacionales.

jmorales@uv.mx

\section{Roberto Lagunes-Córdoba}

Instituto de Investigaciones Psicológicas, Universidad Veracruzana, México

Licenciado en Psicología por la Universidad Veracruzana, Doctor en Neurociencias por el Centro de Investigación y de Estudios Avanzados. Investigador titular de tiempo completo, miembro del Sistema Nacional de Investigadores en México.

rlc.academico@yahoo.com.mx

\section{Agradecimientos}

Por su participación en el proceso de validación de constructo, los autores agradecen a Eleonora Vivas, Yolanda Campos Uscanga y Carlos Escorza Santos; de igual manera se agradece la colaboración en el proceso de validación de contenido a Verónica Patricia Demeneghi Marini, José Roberto Gómez Cruz y Carlos Escorza Santos. 